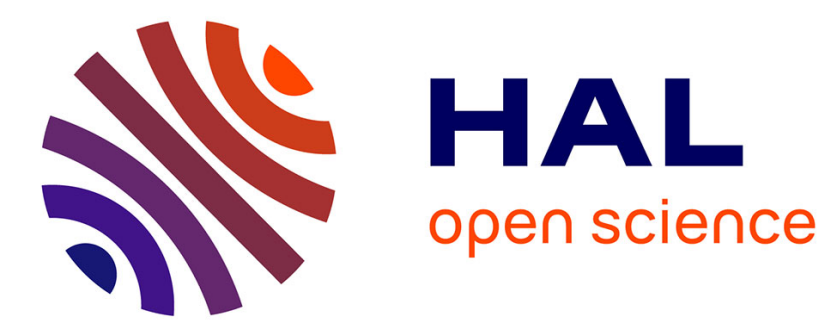

\title{
Prediction of infarct localization from myocardial deformation
}

\author{
Nicolas Duchateau, Maxime Sermesant
}

\section{To cite this version:}

Nicolas Duchateau, Maxime Sermesant. Prediction of infarct localization from myocardial deformation. Statistical Atlases and Computational Modeling of the Heart (STACOM 2015), Oct 2015, Munich, Germany. 10.1007/978-3-319-28712-6_6 . hal-01208019v2

\section{HAL Id: hal-01208019 https://inria.hal.science/hal-01208019v2}

Submitted on 27 Oct 2015

HAL is a multi-disciplinary open access archive for the deposit and dissemination of scientific research documents, whether they are published or not. The documents may come from teaching and research institutions in France or abroad, or from public or private research centers.
L'archive ouverte pluridisciplinaire HAL, est destinée au dépôt et à la diffusion de documents scientifiques de niveau recherche, publiés ou non, émanant des établissements d'enseignement et de recherche français ou étrangers, des laboratoires publics ou privés. 


\title{
Prediction of infarct localization from myocardial deformation
}

\author{
Nicolas Duchateau and Maxime Sermesant \\ Asclepios Research Project, INRIA Sophia Antipolis, France
}

\begin{abstract}
We propose a novel framework to predict the location of a myocardial infarct from local wall deformation data. Non-linear dimensionality reduction is used to estimate the Euclidean space of coordinates encoding deformation patterns. The infarct location of a new subject is inferred by two consecutive interpolations, formulated as multiscale kernel regressions. They consist in (i) finding the low-dimensional coordinates associated to the measured deformation pattern, and (ii) estimating the possible infarct location associated to these coordinates. These concepts were tested on a database of 500 synthetic cases generated from a realistic electromechanical model of the two ventricles. The database consisted of infarcts of random extent, shape, and location overlapping the whole left-anterior-descending coronary territory. We demonstrate that our method is accurate and significantly overcomes the limitations of the clinically-used thresholding of the deformation patterns (average area under the ROC curve of $0.992 \pm 0.011$ vs. $0.812 \pm 0.124, \mathrm{p}<0.001)$.
\end{abstract}

\section{Introduction}

In clinical routine, imaging of the heart often aims at evaluating the local cardiac tissue viability. A decrease in this viability directly affects the electrical propagation and the muscle contraction, and therefore hampers the resulting cardiac function. However, the transfer function linking the local deformation to the tissue viability is complex, due to advanced physiological interactions between the muscle and the blood, the fibers arrangement, or the influence of the neighboring segments or the opposite wall. Late-enhancement imaging is generally accepted as ground truth to localize the regions with scarred tissue $[8,9]$. However, this modality is costly and requires the injection of a contrast agent. Moreover its post-processing is still challenging due to the limited contrast and number of acquired slices. On the other hand, 3D echocardiography is non-ionizing and cheaper, and allows the quantification of local wall deformation (myocardial strain). Cardiologists use it in a daily practice to assess the local tissue viability. Nonetheless, the localization of infarct by thresholding deformation patterns [9] is inaccurate, as the optimal threshold depends on the infarct position and grade, and the relationship between these two parameters is not straightforward.

In this paper, we address this issue by proposing a framework to predict the infarct location from local wall deformation data. While the literature abounds of 

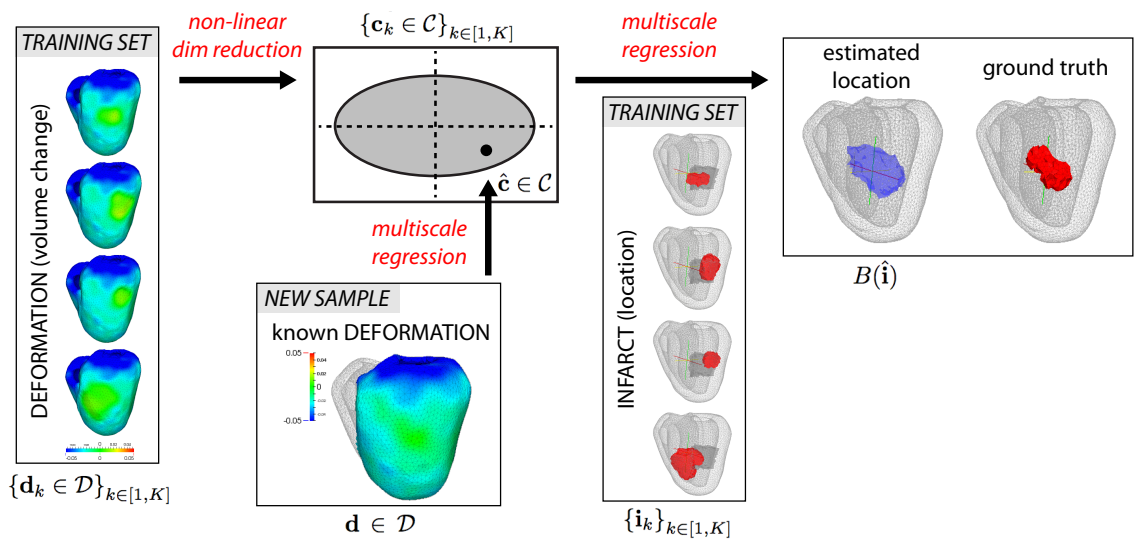

Fig. 1. Proposed method to predict infarct location from local myocardial deformation.

works that threshold the deformation data, our work is novel in designing a more advanced and accurate prediction strategy. Several works investigated variables inference for different cardiac applications, such as the reconstruction of fibers architecture from cardiac shape [5] or to relate cardiac shape remodeling and myocardial infarction [12]. However, these works used linear or logistic regression techniques, which are not necessarily suited to compute statistics on cardiac motion and deformation patterns. For our concrete application, we preferred a formulation based on spectral embedding [11] and kernel regression [3, 4] against a Bayesian formulation, in order to minimize the amount of a-priori knowledge in our method.

A notable asset of the proposed work consists in the large database of synthetic cases created to test the methods against a large variety of infarct configurations. Indeed, in such a framework, the transfer function between the model parameters of healthy/damaged tissue and the local deformation is fully controlled, a requisite to test the methods accuracy on ground truth data. Our experiments show that the prediction of infarct location with our method is significantly more accurate than the clinically-used techniques.

\section{Methods}

In the following, we denote $\left\{\left(\mathbf{d}_{k}, \mathbf{i}_{k}\right)\right\}_{k \in[1, K]}$ the pairs of local deformation and infarct position data for the set of $K$ training samples. This information is available at each point of the volumetric mesh for each subject, and each of these two parameters is treated as a column vector. In the present work, the infarct position is defined as a binary value $0 / 1$ at each vertex. The relative change in the cell volume with respect to the beginning of the cycle is used as a surrogate for local deformation. A single scalar value is therefore associated to the deformation at each point of the mesh. This was preferred over more advanced 
measures of local deformation such as the strain tensor, which would require more advanced statistics. However, using scalar deformation data does not limit the concepts demonstrated in the present work: the observed deformation patterns still reflect local infarct (Figs.1 and 4), and focus is kept on the core of the inverse problem.

\subsection{Non-linear dimensionality reduction (training set)}

The (high-dimensional) local deformation data $\left\{\mathbf{d}_{k} \in \mathcal{D}\right\}_{k \in[1, K]}$ are first mapped to a Euclidean space of (low-dimensional) coordinates $\left\{\mathbf{c}_{k} \in \mathcal{C}\right\}_{k \in[1, K]}$ by means of standard non-linear dimensionality reduction (Isomap [10]). This assumes that there exists a lower-dimensional manifold that can explain the main variations in such data.

In brief, a nearest-neighbors graph is built for the samples $\left\{\mathbf{d}_{k}\right\}_{k \in[1, K]}$, using the Euclidean distance as metric. Then, the geodesic distance between each pair of samples is approximated as the shortest path connecting them along the graph, and put into an affinity matrix. The set of coordinates $\left\{\mathbf{c}_{k}\right\}_{k \in[1, K]}$ is finally obtained by the diagonalization of a centered version of this geodesic distance matrix.

No restriction is made on the manifold learning technique used. We preferred a non-linear one, as linear operations directly on the deformation data may generate unphysiological patterns $[2,4]$ (e.g. the linear average of two deformation patterns corresponding to two disjoint infarcts would provide a mixed widespread pattern reflecting a larger but less accentuated infarct, instead of the pattern of an infarct of similar grade at an intermediate location). The Isomap algorithm was preferred over other spectral embedding techniques [11] as we assumed that the samples distribution follows a uniform random distribution, in contrast with kernel-based methods more relevant for more clustered distributions.

\subsection{From deformation patterns to infarct prediction (testing set)}

Given a new case for which only the local deformation $\mathbf{d}$ is known, our method provides a prediction of the infarct position $B(\hat{\mathbf{i}})$. Its estimation is obtained by two consecutive interpolations formulated as kernel regression, and a multiscale strategy to prevent from artifacts due to non-uniformities in the density of the samples. An overview of the processing pipeline is given in Fig.1.

From deformation patterns to low-dimensional coordinates Given a new deformation pattern $\mathbf{d} \in \mathcal{D}$, a single-scale formulation would compute its corresponding coordinates $\hat{\mathbf{c}} \in \mathcal{C}$ as:

$$
\hat{\mathbf{c}}=\sum_{k=1}^{K} k_{\mathcal{D}}\left(\mathbf{d}, \mathbf{d}_{k}\right) \cdot \mathbf{a}_{k} .
$$


Here $\mathbf{a}_{k}$ is the $k$-th column of the matrix $\left(\mathbf{K}_{\mathcal{D}}+\frac{1}{\gamma_{\mathcal{D}}} \mathbf{I}\right)^{-1} \mathbb{C}$, where $\mathbf{I}$ is the identity matrix, $\mathbb{C}=\left(\mathbf{c}_{1}, \ldots, \mathbf{c}_{K}\right)^{T}, \gamma_{\mathcal{D}}$ is a scalar weight balancing the adherence to the data and the smoothness of the interpolation, and $\mathbf{K}_{\mathcal{D}}=\left(k_{\mathcal{D}}\left(\mathbf{d}_{i}, \mathbf{d}_{j}\right)\right)_{(i, j)}$ is a kernel-based affinity matrix between the input samples. The kernel function is defined as $k_{\mathcal{D}}=\exp \left(-\left\|\mathbf{d}_{i}-\mathbf{d}_{j}\right\|^{2} / \sigma_{\mathcal{D}}^{2}\right), \sigma_{\mathcal{D}}$ being its bandwidth.

Detailed explanations of this single-scale process can be found in [2]. The expression in Eq.1 corresponds to the analytical solution of the following inexact matching problem:

$$
\underset{f \in \mathcal{F}}{\operatorname{argmin}}\left(\frac{1}{2}\|f\|_{\mathcal{F}}^{2}+\frac{\gamma_{\mathcal{D}}}{2} \sum_{k=1}^{K}\left\|f\left(\mathbf{d}_{k}\right)-\mathbf{c}_{k}\right\|^{2}\right),
$$

where $\hat{\mathbf{c}}=f(\mathbf{d})$, and $\|\cdot\|_{\mathcal{F}}$ stands for the norm on the reproducible kernel Hilbert space $\mathcal{F}$ of functions $\mathcal{D} \rightarrow \mathcal{C}$.

In the current application, this process is iterated from large to small scales by dividing the bandwidth $\sigma_{\mathcal{D}}$ by a factor 2 at each iteration $s$, and looking for the remainder function $f-F^{(s-1)}$, where $F^{(s)}$ stands for the $s$-th scale approximation of the original function $f$ [3]. In practice, scales across iterations range from the overall spread of the samples until getting lower than the average density of the samples [3].

From low-dimensional coordinates to infarct prediction An infarct map $\hat{\mathbf{i}} \in \mathcal{I}$ is estimated from the coordinates $\hat{\mathbf{c}}$ using a multiscale regression process similar to the one described in the previous subsection, where $\hat{\mathbf{i}}, \hat{\mathbf{c}}$ and $\mathbf{c}_{k}$ now respectively stand for $\hat{\mathbf{c}}, \mathbf{d}$ and $\mathbf{d}_{k}$ in Eq.1. The interpolating function is now denoted $g$. The infarct map therefore corresponds to $\hat{\mathbf{i}}=g(\hat{\mathbf{c}})=g \circ f(\mathbf{d})$. Data adherence and smoothness are balanced by a weight $\gamma_{\mathcal{C}}$, and the kernel function is $k_{\mathcal{C}}$, of bandwidth $\sigma_{\mathcal{C}}$. In a single-scale formulation, this corresponds to:

$$
\hat{\mathbf{i}}=\sum_{k=1}^{K} k_{\mathcal{C}}\left(\hat{\mathbf{c}}, \mathbf{c}_{k}\right) \cdot \mathbf{b}_{k}
$$

where $\mathbf{b}_{k}$ is the $k$-th column of the matrix $\left(\mathbf{K}_{\mathcal{C}}+\frac{1}{\gamma_{\mathcal{C}}} \mathbf{I}\right)^{-1} \mathbb{I}, \mathbb{I}=\left(\mathbf{i}_{1}, \ldots, \mathbf{i}_{K}\right)^{T}$, and $\mathbf{K}_{\mathcal{C}}=\left(k_{\mathcal{C}}\left(\mathbf{c}_{i}, \mathbf{c}_{j}\right)\right)_{(i, j)}$. This process is also made multiscale, and follows similar rules to the ones used for the estimation of $f$.

Due to this regression process, the values of $\hat{\mathbf{i}}$ at each vertex lie within the continuous $[0,1]$ interval. Thus, the prediction of the infarct position $B(\hat{\mathbf{i}})$ is finally obtained by applying a relevant threshold to $\hat{\mathbf{i}}$ (in our case, previously determined by a ROC analysis, as described in Sec.3.2).

\section{Experiments and results}

\subsection{Dataset}

Infarct generation A database of 500 synthetic cases was generated to evaluate the methods. Starting from a volumetric tetrahedral mesh of the two ven- 

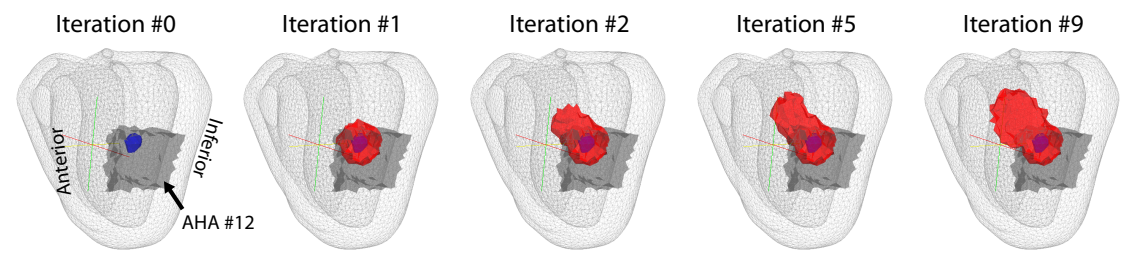

Fig. 2. Iterative generation of an infarcted region (red) with random extent, shape, and location, initiated within the mid-anterolateral segment (black arrow).
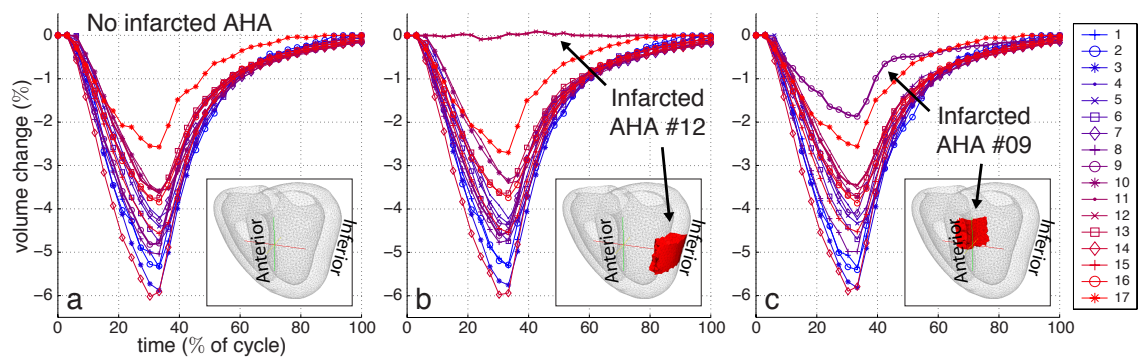

Fig. 3. Average myocardial deformation for each AHA segment in a healthy case $(a)$, and two infarcts in opposite AHA segments ( $b$ and $c$ ).

tricles (46876 cells and 9673 points for the left ventricle [LV], corresponding to a myocardial volume/mass of $173 \mathrm{~mL} / 182 \mathrm{~g}$ ), a fully-connected region corresponding to an infarct of random extent, shape, and location was constructed for each case. The algorithm determined the diseased region iteratively, as illustrated in Fig.2. It first randomly selected a starting point within the leftanterior-descending coronary territory. This specific territory was retained due to its higher prevalence and agreement in its delineation [8]. Note that this only concerns the location of the starting point for the infarct generation algorithm, and that infarcts can spread out of this territory (Fig.4). Further testing should be extended to other coronary territories and different geometries to better evaluate the performance of the method.

A spherical neighborhood of random radius between 2 and $12 \mathrm{~mm}$ was marked as diseased, and a new starting point was randomly selected within this new region. The process was iterated a random number of times (values from 1 to 16). Infarct extents were of $5.2 \pm 2.6 \mathrm{~mL}(3.0 \pm 1.5 \%$ of the $\mathrm{LV}$ myocardium). A total of 400 cases were used as training set and the other 100 served as testing data.

Electromechanical simulation A realistic electromechanical model [6] was then used to simulate the cardiac function along a full cycle of duration $1 \mathrm{~s}$. This model was previously evaluated on invasive clinical data and has a realistic 
behavior. Simulations here use a real anatomical mesh with fibers architecture from an atlas. The contractility and stiffness parameters were altered in the random zone defined by our algorithm to model a local infarct [1]. They partially reflect changes in active force and tissue elasticity. These parameters were retained among many others to limit the training data to manageable amounts, and corresponded to the ones with major influence on the deformation [7]. Border zone was not set to only evaluate the algorithm on binary prediction. Neighbor locations were therefore only passively influenced by the infarct.

Then, local deformation was computed for each tetrahedral cell of the mesh. Figure 3 depicts the deformation values of two infarct configurations at opposite AHA segments, against a healthy case. In this illustrative example, the whole AHA segment was infarcted. Curves represent volume change values averaged over each of the 17 AHA segments of the LV. Notably, deformation patterns are less affected by a mid-anterolateral infarct than by a mid-inferoseptal one, due to the influence of the right ventricle and the surrounding septal regions. Testing our methods on a wide territory of infarct configurations will therefore allow evaluating the sensitivity of the algorithm to marked or moderated alterations of local deformation.

For the sake of simplicity in the infarct prediction process, we limited the input to our algorithm to the deformation data at end-systole (the $\left\{\mathbf{d}_{k} \in \mathcal{D}\right\}_{k \in[1, K]}$ in Sec.2), spatially smoothed by a Gaussian filter of bandwidth $1 \mathrm{~cm}$ to prevent from inconsistencies due to point-cell correspondences and the non-homogeneity of cell sizes and orientation across the whole volumetric mesh. Note that this might lower the accuracy of the infarct prediction near the border of the ground truth location. The use of the deformation data along the whole cycle may add robustness to the results in more complex configurations, where local post-systolic abnormalities may be more marked or in the presence of asynchronous hearts.

Parameters setting The number of nearest neighbors in the Isomap algorithm was set to 5 . The number of dimensions retained for the estimated coordinates was set to 30 . This value corresponded to the limit from which eigenvalues weigh less than $5 \%$ of the first eigenvalue. The scalar weights used in the multiscale regression were determined by a leave-one-out procedure, as the value that minimizes the generalization ability (the reconstruction error for samples lying within the range of noise of the training set). Such values were of $\gamma_{\mathcal{D}}=\gamma_{\mathcal{C}}=1$.

\subsection{Results}

Representative examples of the outputs of our method are shown in Fig.4, to compare with the thresholding of the deformation data from the same cases, in Fig.5 (animated version available as Supplementary Material ${ }^{1}$ ). The latter notably failed on the transmurality of the infarct location. Qualitatively, our method correctly predicted the infarct location, even for infarcts of small size,

${ }^{1}$ http://www-sop.inria.fr/asclepios/docs/TestCasesThresh.zip 


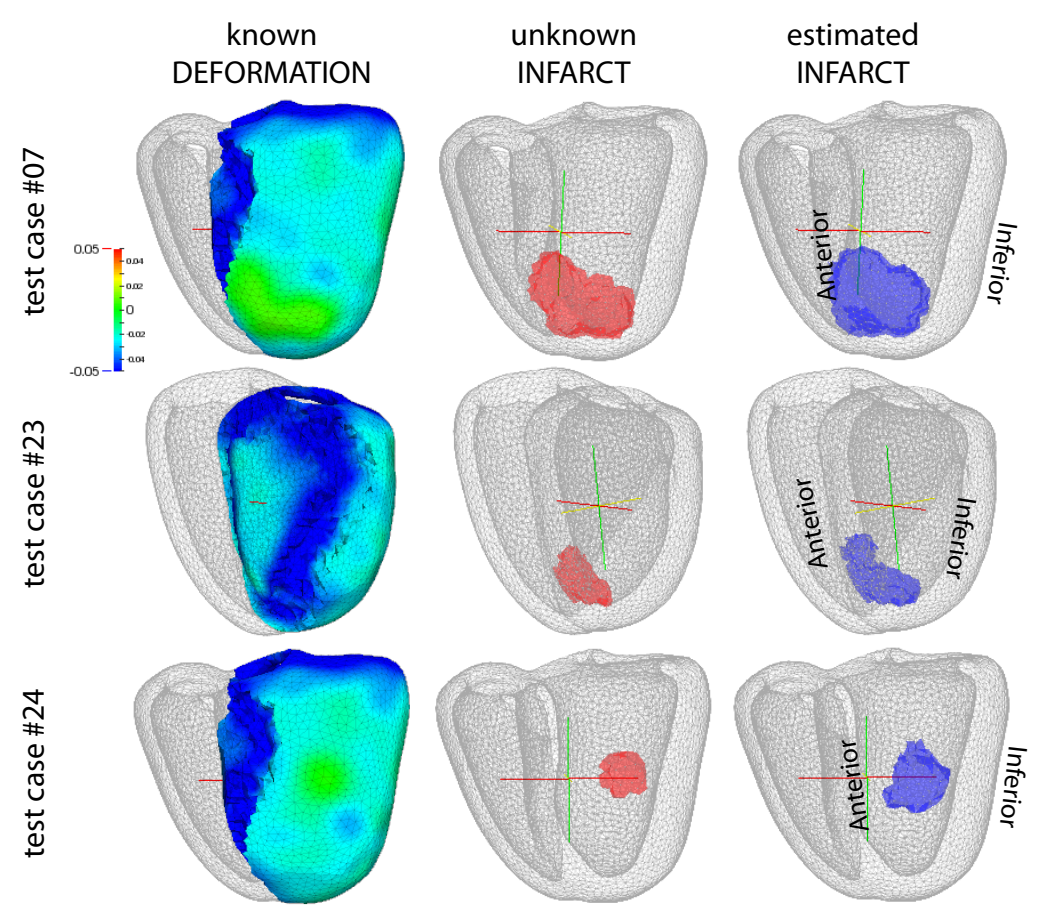

Fig. 4. Examples of myocardial deformation pattern, ground truth infarct location, and estimated infarct location.

location internal to the myocardium, and reduced effect on the deformation curves (e.g. infarcts closer to the septum).

ROC analysis On each case, a ROC analysis was used to determine the optimal threshold leading to the infarct prediction $B(\hat{\mathbf{i}})$ from the infarct map $\hat{\mathbf{i}} \in \mathcal{I}$ (Fig.6a). A similar process was applied for the direct thresholding of the deformation data $\mathbf{d} \in \mathcal{D}$ (Fig.6b). These thresholds were defined as the average of the optimal thresholds for each individual case.

The performance of directly thresholding the deformation was rather poor in terms of sensitivity and specificity (average area under the curve: $0.812 \pm 0.124$ ). Some cases even led to a ROC curve worse than randomly selecting points of the mesh (the diagonal line). Our method significantly outperformed this technique in all cases (average area under the curve: 0.992 $\pm 0.011, \mathrm{p}<0.001$ ).

\section{Conclusion}

We presented a method to predict the location of a myocardial infarct from local deformation patterns. This approach is novel and contrasts with the simpler and 

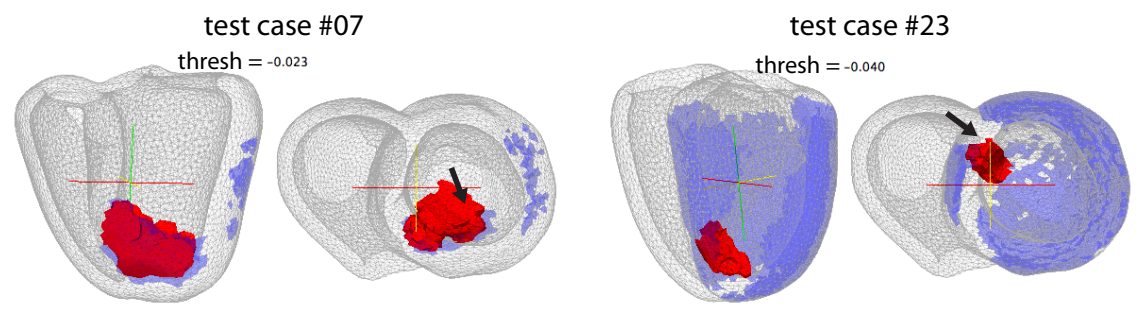

Fig. 5. Ground truth infarct location (red) against the thresholding of the deformation patterns (blue) for the cases shown in Fig.4. Animated version available as Supplementary Material ${ }^{1}$.
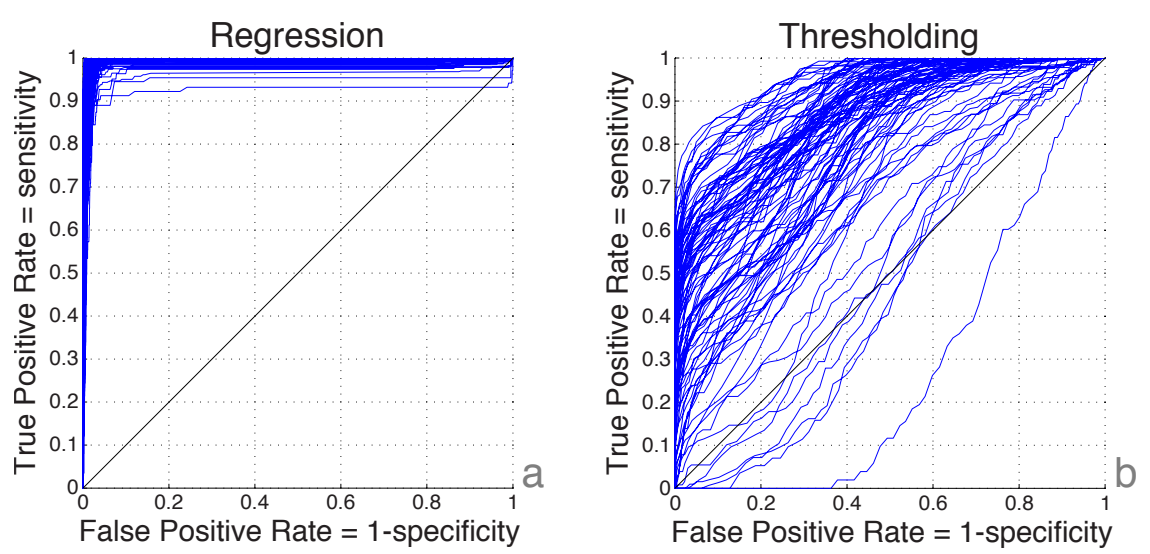

Fig. 6. ROC analysis of the tested cases comparing our method $(a)$ to the thresholding of the deformation patterns $(b)$.

clinically-used thresholding of the deformation patterns. Notably, our method significantly outperformed this technique. A notable asset of the proposed work also resides in the large database used to test the methods, made of synthetic cases with infarcts of random extent, shape, and location. We are currently collecting a database of 3D echocardiographic sequences and late-enhancement images to extend the evaluation of our method to real data. This will allow evaluating the scalability of the method towards the use of different heart geometries, more complex deformation patterns, and possibly less contrasted local changes in the deformation data to the acquisition and post-processing of ultrasound sequences.

Acknowledgements. The authors acknowledge the European Union 7th Framework Programme (VP2HF: FP7-2013-611823) and the European Research Council (MedYMA: ERC-AdG-2011-291080). They also thank their colleagues R. Mollero and S. Giffard-Roisin for their support on practical aspects of the SOFA simulations, and their 
collaborators M. De Craene (Philips Suresnes, France) and E. Saloux (CHU Caen, France) for discussions on these concepts.

\section{References}

1. M Alessandrini, M De Craene, O Bernard, et al. A pipeline for the generation of realistic 3D synthetic echocardiographic sequences: methodology and open-access database. IEEE Transactions on Medical Imaging, 2015. In press.

2. N Duchateau, M De Craene, G Piella, et al. Constrained manifold learning for the characterization of pathological deviations from normality. Medical Image Analysis, 16:1532-49, 2012.

3. N Duchateau, M De Craene, M Sitges, et al. Adaptation of multiscale function extension to inexact matching. Application to the mapping of individuals to a learnt manifold. In SEE-GSI, LNCS 8085, pages 578-86, 2013.

4. S Gerber, T Tasdizen, P Fletcher, et al. Manifold modeling for brain population analysis. Medical Image Analysis, 14:643-53, 2010.

5. K Lekadir, C Hoogendoorn, M Pereanez, et al. Statistical personalization of ventricular fiber orientation using shape predictors. IEEE Transactions on Medical Imaging, 33:882-90, 2014.

6. S Marchesseau, H Delingette, M Sermesant, et al. Personalization of a cardiac electromechanical model using reduced order unscented Kalman filtering from regional volumes. Medical Image Analysis, 17:816-29, 2013.

7. S Marchesseau, H Delingette, M Sermesant, M Sorine, K Rhode, S Duckett, C Rinaldi, R Razavi, and N Ayache. Preliminary specificity study of the BestelClement-Sorine electromechanical model of the heart using parameter calibration from medical images. J Mech Behav Biomed Mater, 20:259-71, 2013.

8. J Ortiz-Perez, J Rodriguez, S Meyers, et al. Correspondence between the 17segment model and coronary arterial anatomy using contrast-enhanced cardiac magnetic resonance imaging. JACC Cardiovascular Imaging, 1:282-92, 2008.

9. B Sjøli, S Ørn, B Grenne, et al. Diagnostic capability and reproducibility of strain by Doppler and by speckle tracking in patients with acute myocardial infarction. JACC Cardiovascular Imaging, 2:24-33, 2009.

10. J Tenenbaum, V De Silva, and J Langford. A global geometric framework for nonlinear dimensionality reduction. Science, 290:2319-23, 2000.

11. S Yan, D Xu, B Zhang, et al. Graph embedding and extensions: a general framework for dimensionality reduction. IEEE Transactions on Pattern Analysis and Machine Intelligence, 29:40-51, 2007.

12. X Zhang, B Cowan, D Bluemke, et al. Atlas-based quantification of cardiac remodeling due to myocardial infarction. PLoS One, 9:e110243, 2014. 